
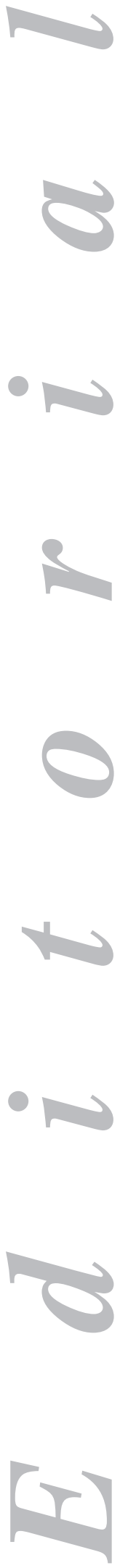

\section{Enfermedad celiaca y pancreatitis: ¿asociación casual o causal?}

La enfermedad celiaca (EC) en una enteropatía de perfil autoinmune mediada por linfocitos $\mathrm{T}$ y desencadenada por la ingesta de gluten que afecta a sujetos predispuestos genéticamente. En la actualidad puede considerarse una enfermedad frecuente que además despierta un notable interés editorial en nuestro país $(1,2)$. Se estima que la prevalencia de la EC puede alcanzar hasta un $1 \%$ de la población general occidental (3). En una reciente publicación del Ministerio de Sanidad se señala una prevalencia en España de 1/118 en niños y de 1/389 en adultos (4).

La aproximación diagnóstica a la EC pasa por la sospecha clínica basada en síntomas y signos, la existencia de un sustrato genético predisponente, la presencia de serología positiva y la comprobación histológica de una lesión intestinal característica.

En la forma clásica de presentación de la EC dominan los síntomas gastrointestinales, mientras que en las variantes atípicas lo hacen los no gastrointestinales y las formas monosintomáticas u oligosintomáticas. Siguiendo la conocida teoría del iceberg de la EC, en su cúspide estaría la forma clásica, por debajo de esta variante se sitúan las formas pauci- u oligosintomáticas, y en la base estarían las formas silentes, en las que existe lesión intestinal característica sin expresión sintomática. Estas serían las dianas del diagnóstico clínico. Aún quedarían por describir dos variantes de dudosa significación clínica: la EC latente, caracterizada por presentar sustrato genético positivo y serología en general también positiva, pero ausencia de lesión histológica y la EC potencial en la que tan sólo sería demostrable una predisposición genética, sin histología positiva y con pruebas serológicas casi siempre negativas. Además, un factor adicional de confusión es que las características clínicas de estas dos últimas variantes con frecuencia aparecen entremezcladas en la literatura.

El síntoma digestivo clásico es la diarrea, pero en el adulto puede estar ausente en la mitad de los casos. Los síntomas extraintestinales de la EC son muy numerosos e incluyen retraso del crecimiento y del desarrollo, osteopenia, osteoporosis, artritis, fracturas patológicas, dolores óseos y musculares generalizados, anemia ferropénica, leucopenia, trombopenia, hemorragia por hipoprotrombinemia, edemas maleolares por hipoalbuminemia, calambres musculares y tetania por hipocalcemia, trastornos reproductivos (menarquia tardía, amenorrea, hipermenorrea, menopausia precoz, abortos espontáneos, infertilidad, impotencia, morbilidad neonatal aumentada), depresión, ansiedad, irritabilidad, trastornos del sueño: insomnio, hipersomnia, astenia acentuada, persistente e inmotivada, fibromialgia, hipoesplenismo, hepatitis reactiva (hipertransaminasemia idiopática), estomatitis aftosa recidivante, hipoplasia del esmalte dentario, dermatitis herpetiforme y polineuropatías (5). Además debe tenerse en cuenta una serie de entidades clínicas, cuyos enfermos presentan un riesgo mayor al de la población general para presentar EC. La guía de práctica clíni- 
ca sobre EC de la Organización Mundial de Gastroenterología (OMG) sugiere que debe investigarse esta entidad en pacientes con anemia ferropénica, deficiencia de ácido fólico y vitamina B12, osteoporosis prematura y osteomalacia, síndrome de Down, hipertransaminasemia no explicada e hipoalbuminemia y que deben considerarse factores de riesgo la diabetes mellitus tipo I, la enfermedad tiroidea autoinmune, el síndrome de Sjögren, la pérdida de peso inexplicada, el retraso puberal y el síndrome de Turner, la deficiencia selectiva de IgA, la neuropatía periférica, el síndrome de intestino irritable, la colitis linfocítica, así como ser familiar de primer y segundo grado de pacientes celiacos (6).

El diagnóstico serológico de EC más aceptado en la actualidad se basa en la determinación de anticuerpos antitransglutaminasa $\operatorname{IgA}$, que posee una especificidad superior al $95 \%$ y sensibilidad en el rango del 90-96\% (7). Se recomienda determinar en paralelo la cifra total de $\operatorname{IgA}$, ya que es posible la coexistencia de EC y déficit de IgA hasta en un $20 \%$ de casos. Si se da esta circunstancia debe procederse a la determinación de anticuerpos antitransglutaminasa $\operatorname{IgG}$ y antiendomisio también IgG para evitar falsos negativos (8).

El uso de los marcadores genéticos de predisposición HLA-DQ2 y HLA-DQ8 no suele ser necesario para el diagnóstico. Su valor reside en la posibilidad de excluir el diagnóstico en caso de negatividad, ya que el $90 \%$ de los celiacos son HLA-DQ2 positivos, frente a un $20-30 \%$ de la población general. El resto de enfermos con EC expresan variantes alélicas HLA-DQ8 o un solo alelo HLA-DQ2, de forma que el valor predictivo negativo de ambas determinaciones es superior al 99\% $(4,9)$.

Desde una perspectiva estricta de probabilidad postprueba, la coexistencia de una positividad serólogica y de un cuadro clínico compatible podría ser suficiente para establecer el diagnóstico de EC. No obstante, la biopsia intestinal tiene un importante sentido clínico pues aumenta nuestra capacidad de integración del diagnóstico en situaciones atípicas, justificando mejor la implantación del tratamiento basado en la restricción dietética completa del gluten. La toma de biopsias adecuadas y múltiples en duodeno, una en bulbo y otras cuatro en diferentes cuadrantes y niveles de la segunda porción permite obtener material concluyente para el diagnóstico en el práctico $100 \%$ de los casos $(10,11)$. Tal como es conocido las biopsias pueden ser clasificadas en 5 niveles o estadios descritos por Marsh: Marsh 0 (mucosa preinfiltrativa); Marsh 1 (incremento en el número de linfocitos intraepiteliales); Marsh 2 (hiperplasia de criptas); Marsh 3 (atrofia vellositaria [3a] parcial, [3b] subtotal, [3c] total); Marsh 4 (hipoplasia de criptas) (12).

La OMG considera que la negatividad conjunta de serología e histología excluye la enfermedad, mientras que una serología positiva y una histología característica la confirman. Ante una serología positiva y una histología negativa recomienda repetir la serología en dos años y seguir entretanto al paciente. La presencia de histología positiva y serología negativa obligan a descartar otras causas de enteritis. Si estas otras causas pueden ser razonablemente rechazadas se sugiere iniciar tratamiento con dieta sin gluten, y evaluar respuesta clínica e histológica. En estos casos dudosos la ausencia de HLA-DQ2 o HLA-DQ8 apoyaría fuertemente la exclusión de la enfermedad celiaca como causa (6).

De lo hasta aquí comentado se deduce que la EC celiaca debe ser sospechada en múltiples situaciones además de en las formas clásicas, pero sólo diagnosticada si las pruebas diagnósticas serológicas e histológicas lo permiten. Esta es la posición coherente con la evidencia consolidada disponible hasta ahora. Sin embargo, algunos grupos clínicos defienden un punto de vista más abierto al diagnóstico de EC aún con negatividad de serología y/o histología. Afirman que no es infrecuente que 
en el adulto la serología pueda ser negativa, o que deben ser tenidos en cuenta niveles de corte para los anticuerpos por debajo de lo marcado por los laboratorios de referencia. Respecto de la histología señalan que la afectación parcheada puede justificar resultados negativos de la biopsia. En torno a este problema, Rodrigo y cols. (5), en su excelente revisión publicada este mismo año indican textualmente: "Lo más importante para llegar al diagnóstico de la EC es que el médico piense en su posible existencia y la incluya en el diagnóstico diferencial teniendo en cuenta la amplia variedad de rasgos clínicos que puede presentar la enfermedad, que es ciertamente muy polimorfa. Conviene recordar que no hay ninguna prueba que sea capaz de diagnosticarla por sí sola de forma concluyente o, por el contrario, excluirla de forma definitiva". Por ello entre las estrategias diagnósticas que recomiendan incluyen la valoración de la respuesta clínica e histológica tras la retirada del gluten en todos aquellos casos en los que exista sospecha clínica, aun sin criterios diagnósticos serológicos o histológicos claros.

La posición de aceptar que los pacientes serológicamente negativos pueden de hecho tener una EC ha sido también recogida por nuestro Ministerio de Sanidad y Consumo a la hora de diseñar la estrategia recomendada para la derivación de los pacientes sospechosos de EC desde atención primaria a las consultas de especialidad (4). El problema de este planteamiento es dar carta de cuasi certeza a una situación de baja probabilidad real de enfermedad. Se defiende que es posible la existencia de sujetos con serología negativa y lesiones histológicas muy leves que en realidad presenten EC. Esta posición es razonable si se acepta que el rendimiento de la serología es deficiente en los umbrales clínicos de la enfermedad. Esta actitud favorece el incremento diagnóstico de EC en situaciones frontera, pero al no subclasificar estos casos como especiales de hecho modifica los datos referentes a la EC de significación clínica cierta. La situación se complica aún más si se aceptan como clínicas compatibles con EC una lista extendida de problemas y situaciones de riesgo en las que la EC puede estar o no presente. Afirmar, por ejemplo, que puede darse por cierta la presencia de una EC serológicamente negativa con un Marsh 1 en un paciente HLA-DQ2 positivo con una polineuropatía aislada, parece excesivo salvo que nos refiramos estrictamente a variantes de significación clínica incierta. En esta línea sólo la ausencia de patrones de susceptibilidad genética parece ser capaz de negar la EC, y aun esto empieza a ser discutido en algún caso extremo. El límite llegaría si se decidiera que todo cuadro compatible puede ser aceptado como EC, aún en ausencia de serología, histología y susceptibilidad genética siempre que exista respuesta clínica a la retirada del gluten. Aún sin llegar a este punto, la ganancia en términos de beneficio individual potencial del paciente, cuando se le ofrece un tratamiento sin un diagnóstico aceptable desde la evidencia, se paga con la imprecisión diagnóstica. Es decir, debemos ser cuidadosos al extender el espectro diagnóstico en términos de práctica clínica individual a las series de pacientes pues los errores de mala clasificación pueden ser muy notables. En estas series sería mejor hablar de EC confirmada de acuerdo a los patrones aceptados y de EC posible o probable para el resto de casos, o en su caso de enteropatía celiaca de significado incierto. Esto es así porque la respuesta clínica observada fuera de ensayos clínicos no puede científicamente atribuirse sin margen de posible error al tratamiento realizado, ya que múltiples circunstancias diferentes a la dieta sin gluten, no controladas por la ausencia de asignación aleatoria, pueden modificar esa respuesta, incluyendo la propia historia natural del proceso clínico bajo observación.

En este difícil contexto Rodrigo y cols. vuelven a realizar una notable contribución al conocimiento de la EC dentro de este mismo número de la Revista Española 
de Enfermedades Digestivas al abordar la eventual relación entre pancreatitis y EC. No es una casualidad que también en este número pueda consultarse una brillante conferencia clínico-patológica que ilustra esta misma posible relación. Rodrigo y cols. a lo largo de todo su trabajo no utilizan el término EC sino el de enteropatía por gluten (EG), pero no marcan diferencias de clasificación explícitas con la EC, término que sí emplea el mismo grupo en la ya citada revisión previa, por lo que entendemos que lo consideran sinónimo. En el interesante artículo que ahora nos ocupa exponen los hallazgos de una serie prospectiva de pacientes con pancreatitis aguda recidivante. En la introducción de su trabajo los autores señalan estudio clásicos, publicados entre 1963 y 1972, en los que se postula una asociación de la pancreatitis crónica y la insuficiencia pancreática con la EG. Abrazan la hipótesis de que los trastornos biliares y pancreáticos en la EG pueden quizás ser atribuidos a los cambios inflamatorios duodenales y del área papilar. Para avanzar en el conocimiento de la relación entre páncreas y la EC, o la EG, nos presentan el análisis de una serie prospectiva de 185 pacientes con pancreatitis aguda (PA), de los que 40 cumplen el criterio de pancreatitis recurrente o recidivante, al haber presentado más de un episodio. A estos 40 sujetos se les aplica un protocolo de diagnóstico de EG serológico, histológico y genético. Los criterios diagnósticos de EG en este trabajo se describen de la siguiente manera: "Fueron diagnosticados de enteropatía sensible al gluten (EG), todos aquellos pacientes que presentaban rasgos clínicos y/o analíticos compatibles, junto con la presencia de marcadores genéticos de susceptibilidad y/o marcadores serológicos positivos (TGt elevada) y/o lesiones histológicas duodenales compatibles y/o respuesta a la dieta sin gluten de forma mantenida, más de seis meses. Dentro de ella se incluyen las denominadas formas latente y potencial de la enfermedad celiaca (EC)". El resultado es que 34 de estos 40 sujetos (85\%) reunían al menos uno de los cuatro criterios. La única diferencia significativa entre los sujetos que recidivaron y presentaron los criterios de EG (34) y el resto de la serie de PA, recidivante o no, fue una menor proporción de litiasis biliar entre los diagnosticados de EG. Este hecho puede explicarse por el adecuado manejo de la PA de origen biliar tras el primer episodio. En las tablas II y III Rodrigo y cols. señalan la proporción de pacientes que cumplen algunos de los criterios diagnósticos. Destaca que aproximadamente la mitad de los pacientes eran HLA-DQ2 o HLA-DQ8 negativos, y más del $40 \%$ Marsh 0 . Sólo el $9 \%$ fueron serológicamente positivos y nada más que 2 pacientes $(6 \%)$ presentaron atrofia vellositaria clara (Marsh 3), mientras que otro $30 \%$ presentaron un estadio Marsh 2. En el texto nos informan de un $88 \%$ de respuesta a la retirada del gluten en el sentido de ausencia de nueva recidiva al año, aunque no se ofrecen más detalles. Ya en la discusión, los autores aportan la hasta ahora escasa literatura producida en los últimos años que asocia pancreatitis y EC, destacando la cohorte basada en registro que demuestra un riesgo aumentado de pancreatitis aguda y crónica entre los sujetos diagnosticados de EC en la edad adulta (13). También señalan la publicación de Patel y cols. en la que se postula un mecanismo obstructivo ligado a estenosis papilar en la EC para algunos de los casos de pancreatitis recurrente (14). Finalmente se comenta otro original en el que se postula que la asociación entre EC y pancreatitis cabría entenderla más bien en el contexto de un proceso autoinmune (15). La recomendación que los autores realizan a modo de conclusión es la de tener en cuenta la EG, o la EC, a la hora de establecer posibles causas para los casos de pancreatitis aguda recidivante. A la vista de la información aportada, esta recomendación debe tenerse en cuenta, aunque tal como este editorial defiende debería hacerse en el marco prudente de los diagnósticos de EG o EC de incierto significado, dada la laxitud de los criterios diagnósticos aplica- 
dos. Los mecanismos que sustentarían esta eventual asociación causal no están tampoco claros en esta serie pues los cambios histológicos encontrados no parecen justificar mecanismos obstructivos, y no se aporta información sobre posibles variantes autoinmunes. El profundo aprecio por la aportación de Rodrigo y cols. es la de ofrecer la oportunidad de trabajar con ahínco en esta línea, tanto en el seno de su grupo como de otros igualmente interesados en este tema, quizás generando estudios casocontrol, en los que aplicando la misma metodología diagnóstica a casos y controles sea posible establecer si verdaderamente existe asociación causal y determinar su magnitud, sin olvidar sin duda otros estudios de perfil clínicos o sobre mecanismos de enfermedad.

\section{J. Egea Valenzuela y F. Carballo Álvarez \\ Servicio de Medicina de Aparato Digestivo. Hospital Universitario Virgen de la Arrixaca. Murcia}

\section{Bibliografía}

1. Rodrigo Sáez L. Celiac disease in the adult. Rev Esp Enferm Dig 2006; 98(6): 397-407.

2. Casellas Jorda F, Malagelada Benapres JR. Chiaroscuros of celiac disease. Rev Esp Enferm Dig 2008; 100(1): 1-4.

3. Dube C, Rostom A, Sy R, Cranney A, Saloojee N, Garritty C, et al. The prevalence of celiac disease in average-risk and at-risk Western European populations: a systematic review. Gastroenterology 2005; 128(4 Supl. 1): S57-67.

4. Diagnóstico precoz de la enfermedad celiaca. Ministerio de Sanidad y Consumo; 2008.

5. Rodrigo L, Garrote JA, Vivas S. Celiac disease. Med Clin 2008; 131(7): 264-70.

6. WGO practical guidelines. Celiac disease. World Gastroenterology Organisation; 2007.

7. AGA Institute Medical Position Statement on the Diagnosis and Management of Celiac Disease. Gastroenterology 2006; 131(6): 1977-80.

8. Rostom A, Murray JA, Kagnoff MF. American Gastroenterological Association (AGA). Institute technical review on the diagnosis and management of celiac disease. Gastroenterology 2006; 131(6): 1981-2002.

9. Hadithi M, von Blomberg BM, Crusius JB, Bloemena E, Kostense PJ, Meijer JW, et al. Accuracy of serologic tests and HLA-DQ typing for diagnosing celiac disease. Ann Intern Med 2007; 147(5): 294-302.

10. Hopper AD, Cross SS, Sanders DS. Patchy villous atrophy in adult patients with suspected gluten-sensitive enteropathy: is a multiple duodenal biopsy strategy appropriate? Endoscopy 2008; 40(3): 219-24.

11. Pais WP, Duerksen DR, Pettigrew NM, Bernstein CN. How many duodenal biopsy specimens are required to make a diagnosis of celiac disease? Gastrointestinal Endoscopy 2008; 67(7): 1082-7.

12. Marsh MN. Gluten, major histocompatibility complex, and the small intestine. A molecular and immunobiologic approach to the spectrum of gluten sensitivity ('celiac sprue'). Gastroenterology 1992; 102(1): 330-54

13. Ludvigsson JF, Montgomery SM, Ekbom A. Risk of pancreatitis in 14,000 individuals with celiac disease. Clin Gastroenterol Hepatol 2007; 5(11): 1347-53.

14. Patel RS, Johlin FC Jr, Murray JA. Celiac disease and recurrent pancreatitis. Gastrointestinal Endoscopy 1999; 50(6): 823-7.

15. Leeds JS, Sanders DS. Risk of pancreatitis in patients with celiac disease: is autoimmune pancreatitis a biologically plausible mechanism? Clin Gastroenterol Hepatol 2008; 6(8): 951 (author reply). 